\title{
Regio- and stereoselective synthesis of 1-aryl-1-thio-2- thiophosphinylethene derivatives via a radical process
}

\section{$\operatorname{AUTHOR}(\mathrm{S})$ :}

SATO, Akinori; YORIMITSU, Hideki; OSHIMA, Koichiro

\section{CITATION:}

SATO, Akinori ...[et al]. Regio- and stereoselective synthesis of 1-aryl-1-thio-2-

thio phosphinylethene derivatives via a radical process. Tetrahedron 2009, 65(8): 15531558

\section{ISSUE DATE:}

2009-02-21

URL:

http://hdl.handle.net/2433/123413

\section{RIGHT:}

Copyright (C) 2009 Elsevier; This is not the published version. Please cite only the published version.; この論文は出版社版でありません。引用の 際には出版社版をご確認ご利用ください。 


\section{Graphical Abstract}

Regio- and Stereoselective Synthesis of $(E)$-1Leave this area blank for abstract info.

Aryl-1-thio-2-thiophosphinylethene

Derivatives via a Radical Process

Akinori Sato, Hideki Yorimitsu, Koichiro Oshima*

Department of Material Chemistry, Graduate School of Engineering, Kyoto University, Kyoto-daigaku Katsura, Nishikyo-ku, Kyoto 615-8510, Japan

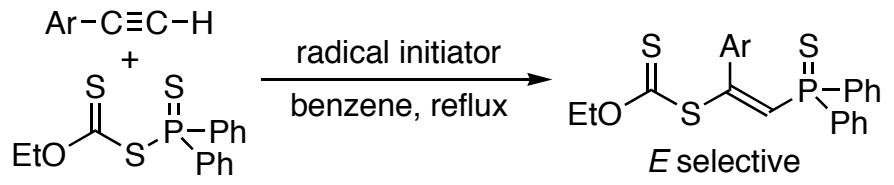




\title{
Regio- and Stereoselective Synthesis of 1-Aryl-1-thio-2- thiophosphinylethene Derivatives via a Radical Process
}

\author{
Akinori Sato, Hideki Yorimitsu, and Koichiro Oshima* \\ Department of Material Chemistry, Graduate School of Engineering, Kyoto University, Kyoto-daigaku Katsura, Nishikyo-ku, Kyoto 615- \\ 8510, Japan
}

\begin{abstract}
Radical additions of $S$-thiophosphinyl dithiocarbonate to terminal aromatic alkynes afford (E)-1-aryl-1-thio-2thiophosphinylethene derivatives regio- and stereoselectively in high yields. The transformations of the products are also described.

(C) 2010 Elsevier Science. All rights reserved
\end{abstract}

\section{Introduction}

Radical additions of heteroatom-heteroatom bonds to alkenes and alkynes are fundamental methods to introduce two heteroatoms to organic molecules in one operation. Among them, radical additions of dicharcogenides to carbon-carbon multiple bonds have been widely investigated. ${ }^{1}$ However, in spite of the increasing utilities of organophosphorus compounds in organic chemistry, ${ }^{2}$ examples of radical additions of phosphorus-heteroatom bonds are limited. ${ }^{3,4}$ Our group recently reported a radical thiophosphination of alkynes with thiophosphines (Scheme $1) .{ }^{4}$ The reaction proceeds through the addition of sulfurcentered radical to the terminal carbon of alkyne followed by the efficient reaction of the resulting vinyl radical with the phosphino group of thiophosphine.

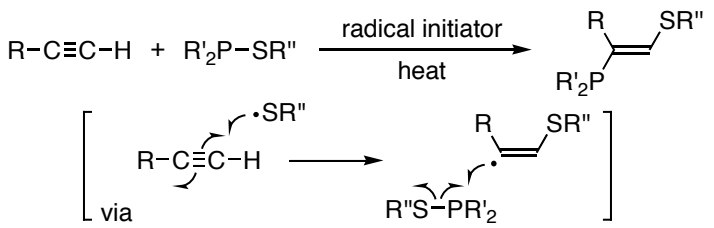

Scheme 1. Our previous work ${ }^{4 b}$
After reporting the reaction shown in Scheme $1,{ }^{4 b}$ we explored another intermolecular radical addition reaction to introduce both phosphorus and sulfur atoms to alkynes with the regioselectivity opposite to the previous reaction. Here we report the synthesis of (E)-1-aryl-1-thio-2thiophosphinylethene derivatives via a radical process using a thiophosphinylated dithiocarbonate. ${ }^{5}$ Stereoselective syntheses of (E)-1-aryl-1-thio-2thiophosphinylethenes are scarcely reported. ${ }^{6}$

\section{Result and discussion}

A mixture of phenylacetylene (1a), $S$ diphenylthiophosphinyl $O$-ethyl dithiocarbonate (2), and a catalytic amount of dilauroyl peroxide (DLP, $\left.\left({ }^{n} \mathrm{C}_{11} \mathrm{H}_{23} \mathrm{CO}_{2}\right)_{2}\right)^{7}$ was heated in benzene at reflux temperature for $4 \mathrm{~h}$ (Table 1, entry 1 ). NMR analysis of the crude mixture indicated the formation of the corresponding adduct, $S$-2-diphenylthiophosphinyl-1-phenylethenyl $O$ ethyl dithiocarbonate (91\%, a single isomer). Silica gel column chromatography followed by gel permeation chromatography (GPC) $)^{8}$ afforded $\mathbf{3 a}$ in $76 \%$ yield. We confirmed by X-ray analysis that the $E$ isomer was exclusively formed.

\footnotetext{
Keywords: Radical reaction, $\mathrm{C}-\mathrm{P}$ bond formation, $\mathrm{C}-\mathrm{S}$ bond formation, dithiocarbonate.

${ }^{*}$ Corresponding authors. Tel.: +81-75383-2441; fax: +81-75383-2438; e-mail: yori@orgrxn.mbox.media.kyoto-u.ac.jp; oshima@orgrxn.mbox.media.kyotou.ac.jp.
} 
This reaction would proceed as follows (Scheme 2). Initially, an undecyl radical, thermally generated from DLP, would attack the sulfur atom of the thiocarbonyl moiety of 2 to generate diphenylthiophosphinoyl radical (A) with the formation of $O$-ethyl $S$-undecyl dithiocarbonate. ${ }^{5}$ The phosphorus-centered radical $\mathbf{A}$ would react with the triple bond of 1a to furnish vinyl radical $\mathbf{B} .^{9}$ An equilibrium would exist between the radicals $\mathbf{B}$ and $\mathbf{B}$ '. However, only radical $\mathbf{B}$ would react with $\mathbf{2}$ to furnish radical $\mathbf{C}$ (vide infra). Finally, fragmentation of radical $\mathbf{C}$ would produce 3a and regenerate the phosphorus-centered radical $\mathbf{A}$ to complete the radical chain.

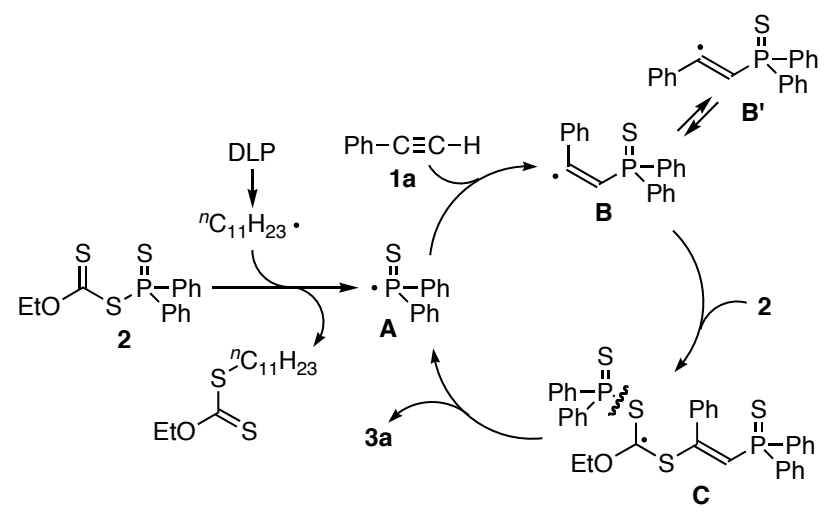

Scheme 2.

The $E$ selectivity of the reaction can be explained as outlined in Scheme 3. Vinyl radical $\mathbf{B}^{\prime}$ would reside in larger population than radical $\mathbf{B}$ in the equilibrium because radical $\mathbf{B}^{\prime}$ has a favorable $E$ configuration. However, the diphenylthiophosphinyl moiety and reagent $\mathbf{2}$ are so large that the steric repulsion between $\mathbf{B}$ ' and $\mathbf{2}$ would prevent the reaction. Hence, only $\mathbf{B}$ can react with $\mathbf{2}$ to form the product 3a exclusively.

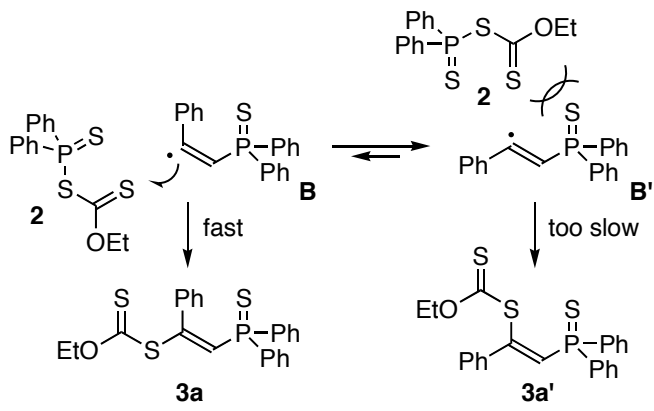

Scheme 3.

A variety of aryl-substituted terminal alkynes underwent the radical addition reaction with complete regio- and stereoselectivity (Table 1 ). The $E$ configurations of $\mathbf{3}$ were deduced from comparing the ${ }^{1} \mathrm{H}$ NMR spectra of $\mathbf{3 b}-\mathbf{3 k}$ with the spectrum of $\mathbf{3 a}$. Both electron-rich and electrondeficient aryl-substituted acetylenes reacted with $\mathbf{2}$ to afford $\mathbf{3}$ in high yields. The addition of $\mathbf{2}$ to sterically hindered alkynes like 1c and 1d proceeded smoothly (entries 3 and 4). Functional groups such as alkoxy (entry 5), keto (entry 6), cyano (entry 7), ester (entry 8), formyl (entry 9), and bromo (entry 10) moieties remained unchanged under the reaction conditions. It is worth noting that a hydroxy group did not affect the reactivity (entry 11). However, attempts to perform addition reactions of $\mathbf{2}$ across 1-dodecyne, trimethylsilylacetylene, methyl propiolate, and 1-phenylpropyne resulted in failure, suffering from low conversion.

Table 1. Addition of $\mathbf{2}$ to various terminal alkynes

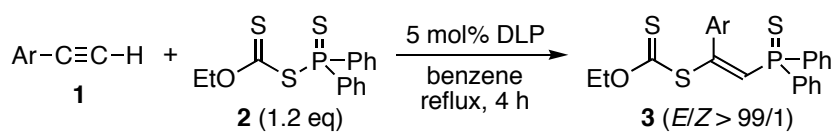

\begin{tabular}{|c|c|c|c|}
\hline Entry & $\mathrm{Ar}$ & Products & $\begin{array}{c}\text { isolated } \\
\text { yield }(\%)\end{array}$ \\
\hline 1 & $\mathrm{Ph}(\mathbf{1 a})$ & $3 \mathbf{a}$ & $76^{\mathrm{a}}(91)^{\mathrm{c}}$ \\
\hline 2 & $4-\mathrm{Me}-\mathrm{C}_{6} \mathrm{H}_{4}(\mathbf{1 b})$ & $\mathbf{3 b}$ & $71^{b}(89)^{c}$ \\
\hline 3 & $2,4,6-\mathrm{Me}_{3}-\mathrm{C}_{6} \mathrm{H}_{2}(\mathbf{1 c})$ & $3 c$ & $83^{\mathrm{a}}$ \\
\hline 4 & 1-naphthyl (1d) & 3d & 73 \\
\hline 5 & $4-\mathrm{MeO}-\mathrm{C}_{6} \mathrm{H}_{4}(\mathbf{1 e})$ & $3 e$ & 88 \\
\hline 6 & 4-Ac- $\mathrm{C}_{6} \mathrm{H}_{4}(\mathbf{1 f})$ & $3 f$ & 92 \\
\hline 7 & $4-\mathrm{NC}-\mathrm{C}_{6} \mathrm{H}_{4}(\mathbf{1 g})$ & $3 g$ & 85 \\
\hline 8 & $4-\mathrm{MeO}_{2} \mathrm{C}-\mathrm{C}_{6} \mathrm{H}_{4}(\mathbf{1 h})$ & $3 h$ & 79 \\
\hline 9 & $4-\mathrm{OHC}-\mathrm{C}_{6} \mathrm{H}_{4}(\mathbf{1 i})$ & $3 \mathbf{i}$ & 88 \\
\hline 10 & $4-\mathrm{Br}-\mathrm{C}_{6} \mathrm{H}_{4}(\mathbf{1} \mathbf{j})$ & $3 \mathbf{j}$ & $87^{\mathrm{b}}$ \\
\hline 11 & $4-\mathrm{HOCH}_{2}-\mathrm{C}_{6} \mathrm{H}_{4}(\mathbf{1 k})$ & $3 \mathbf{k}$ & 88 \\
\hline
\end{tabular}

${ }^{a}$ Yields after purification by GPC. ${ }^{\mathrm{b}}$ Yields after recrystallization.

${ }^{\mathrm{c}}$ Yields in parentheses were determined by ${ }^{31} \mathrm{P}$ NMR.

The reaction of thiophosphinyl dithiocarbonate 2 with $p$ diethynylbenzene (11) efficiently afforded $p$-bis(1-thio-2thiophosphinylethenyl)benzene 31. The double addition proceeded with perfect regio- and stereoselectivity in a very high yield (Scheme 4).<smiles>C#Cc1ccc(C#CC)cc1</smiles>

Scheme 4.

We next attempted to carry out stereospecific transformations of product 3 . It was found that hydrolysis of the dithiocarbonate moieties of $\mathbf{3}$ followed by $S$ alkylation proceeded ${ }^{10}$ in the presence of excess potassium hydroxide and reactive alkyl halides 4 to provide the thiophosphinylated vinyl sulfides $\mathbf{5}$ in excellent yields with retention of configuration (Table 2 ). ${ }^{11}$

Table 2. Hydrolysis of $\mathbf{3}$ followed by alkylation with $\mathbf{4}$

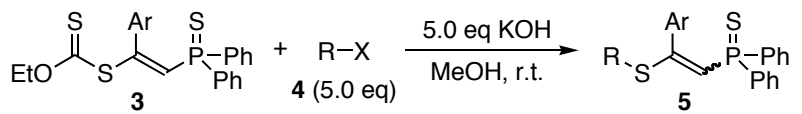




\begin{tabular}{cccccc}
\hline entry & $\mathbf{3}$ & $\mathrm{R}-\mathrm{X}(\mathbf{4})$ & products & $\begin{array}{c}\text { isolated } \\
\text { yield (\%) }\end{array}$ & $E / Z$ \\
\hline 1 & $\mathbf{3 a}$ & $\mathrm{Me}-\mathrm{I}(\mathbf{4 a})$ & $\mathbf{5 a a}$ & 98 & $98 / 2$ \\
2 & $\mathbf{3 a}$ & allyl-Br (4b) & $\mathbf{5 a b}$ & 98 & $98 / 2$ \\
3 & $\mathbf{3 a}$ & $\mathrm{Bn}-\mathrm{Br}(\mathbf{4 c})$ & $\mathbf{5 a c}$ & 88 & $99 / 1$ \\
4 & $\mathbf{3 b}$ & $\mathrm{Me}-\mathrm{I}(\mathbf{4 a})^{\mathrm{a}}$ & $\mathbf{5 b a}$ & 93 & $98 / 2$ \\
\hline
\end{tabular}

\section{Experimental}

\subsection{General}

\subsubsection{Instrumentation}

${ }^{1} \mathrm{H}$ NMR (500 MHz) and ${ }^{13} \mathrm{C}$ NMR (125.7 MHz) spectra were taken on a Varian UNITY INOVA 500 spectrometer and were obtained in $\mathrm{CDCl}_{3}$ with tetramethylsilane as an internal standard. ${ }^{31} \mathrm{P}$ NMR (121.5 MHz) spectra were taken on a Varian MERCURY 300 spectrometer and were obtained in $\mathrm{CDCl}_{3}$ with $85 \% \mathrm{H}_{3} \mathrm{PO}_{4}$ solution as an external standard. Chemical shifts $(\delta)$ are in parts per million relative to tetramethylsilane at $0.0 \mathrm{ppm}$ for ${ }^{1} \mathrm{H}$, relative to $\mathrm{CDCl}_{3}$ at $77.0 \mathrm{ppm}$ for ${ }^{13} \mathrm{C}$, and relative to $\mathrm{H}_{3} \mathrm{PO}_{4}$ at $0.0 \mathrm{ppm}$ for ${ }^{31} \mathrm{P}$. DEPT, $\mathrm{H}-\mathrm{H}$ COSY, H-C COSY, and NOE analyses allowed the assignments of the signals of each compounds. IR spectra were determined on a SHIMADZU FTIR-8200PC spectrometer. Mass spectra were determined on a JEOL Mstation 700 spectrometer. Melting points were determined on Yanaco micro melting point apparatus. TLC analyses were performed on commercial glass plates bearing a $0.25-\mathrm{mm}$ layer of Merck Silica gel $60 \mathrm{~F}_{254}$. Silica gel (Wakogel 200 mesh) was used for column chromatography. Gel permeation chromatography (GPC) was performed by LC-908 (Japan Analytical Industry Ltd., two in-line JAIGEL-2H, toluene, $10 \mathrm{~mL} / \mathrm{min}$, UV and RI detectors). Elemental analyses were carried out at the Elemental Analysis Center of Kyoto University.

\subsubsection{Materials}

Unless otherwise noted, materials obtained from commercial suppliers were used without further purification. Benzene, methanol, CPME, diphenylthiophosphinyl chloride, and potassium hydroxide were purchased from Wako Pure Chemicals. Potassium $O$ ethyl dithiocarbonate and $\mathrm{NiCl}_{2}$ (dppp) were available from TCI. DLP and iodomethane were obtained from Nacalai Tesque. Neat diethylzinc was purchased from Aldrich and was diluted with degassed dry hexane to prepare a $1.0 \mathrm{M}$ solution, which was stored under argon. Benzene was dried over slices of sodium. Alkynes were commercially available or readily prepared by the conventional Sonogashira reactions. $S$-Diphenylthiophosphinyl $O$-ethyl dithiocarbonate (2) was synthesized from potassium $O$ ethyl dithiocarbonate and diphenylthiophosphinyl chloride (vide infra).

\subsection{Experimental Procedures}

\subsubsection{Preparation of $S$-diphenylthiophosphinyl $O$-ethyl dithiocarbonate (2)}

Under argon, diphenylthiophosphinyl chloride $(3.08 \mathrm{~mL}$, $15.0 \mathrm{mmol}$ ) was added to a suspension of potassium $O$ ethyl dithiocarbonate $(2.64 \mathrm{~g}, 16.5 \mathrm{mmol})$ in THF (20.0 $\mathrm{mL}$ ) at ambient temperature. After $4 \mathrm{~h}$, a diluted $\mathrm{NaCl}$ solution was poured into the suspension and the mixture was extracted twice with hexane/EtOAc $=2 / 1$. The organic extracts were dried over $\mathrm{Na}_{2} \mathrm{SO}_{4}$, filtered, and concentrated in vacuo. Silica gel column chromatography (hexane/EtOAc $=20 / 1$ to $10 / 1$ ) afforded $S$ - 
diphenylthiophosphinyl $O$-ethyl dithiocarbonate (2) as a yellow solid in $84 \%$ yield $(4.25 \mathrm{~g}, 12.6 \mathrm{mmol})$.

\subsubsection{General procedure for additions of thiophosphinyl dithiocarbonate 2 to alkynes 1}

Under argon, a mixture of alkyne 1 (0.50 mmol), $S$ diphenylthiophosphinyl $O$-ethyl dithiocarbonate $(2,0.20 \mathrm{~g}$, $0.60 \mathrm{mmol})$, and DLP (0.010 g, $0.025 \mathrm{mmol})$ was heated in boiling benzene $(2.0 \mathrm{~mL})$ for $4 \mathrm{~h}$. After cooled to room temperature, the solvent was removed in vacuo. Silica gel column chromatography (hexane/EtOAc) followed by GPC or recrystallization (if needed) afforded a 1-aryl-1-thio-2thiophosphinylethene derivative $\mathbf{3}$ as a white or yellow solid $(E / Z>99 / 1)$. The $E$ configuration was assigned by $\mathrm{X}-$ ray analysis (See the Caracterization Data of $\mathbf{3 a}$ ).

\subsubsection{Double addition of thiophosphinyl dithiocarbonate 2 to 11}

Under argon, a mixture of $p$-diethynylbenzene (11, $0.063 \mathrm{~g}$, $0.50 \mathrm{mmol}), \quad S$-diphenylthiophosphinyl $O$-ethyl dithiocarbonate $(2,0.51 \mathrm{~g}, 1.5 \mathrm{mmol})$, and DLP $(0.010 \mathrm{~g}$, $0.025 \mathrm{mmol})$ was heated in boiling benzene $(3.0 \mathrm{~mL})$ for 4 h. After cooled to room temperature, the solvent was removed in vacuo. Filtration with EtOAc followed by short column chromatography on silica gel $\left(\mathrm{CHCl}_{3}\right)$ afforded 1,4-bis $\{(E)$-2-diphenylthiophosphinyl-1-

(ethoxythiocarbonylsulfanyl)ethenyl benzene (3I) as a pale yellow solid in $92 \%$ yield $(0.37 \mathrm{~g}, 0.46 \mathrm{mmol})$

\subsubsection{General procedure for transformation of dithiocarbonates 3 to sulfides 5}

Transformation of 3a to 5aa is representative. Under argon, ground potassium hydroxide $(0.056 \mathrm{~g}, 1.0 \mathrm{mmol})$ was added to a suspension of dithiocarbonate 3a $(0.088 \mathrm{~g}, 0.20$ $\mathrm{mmol}$ ) and iodomethane $(4 \mathbf{a}, 0.062 \mathrm{~mL}, 1.0 \mathrm{mmol})$ in methanol $(3.0 \mathrm{~mL})$ at ambient temperature. After being stirred for $30 \mathrm{~min}$, the mixture was quenched with a diluted $\mathrm{NaCl} / \mathrm{NH}_{4} \mathrm{Cl}$ solution and extracted three times with hexane/EtOAc $=1 / 1$. The organic extracts were dried over $\mathrm{Na}_{2} \mathrm{SO}_{4}$, filtered, and concentrated in vacuo. Silica gel column chromatography (hexane/EtOAc $=10 / 1$ ) afforded \{2-diphenylthiophosphinyl-1-(methylthio)ethenyl $\}$ benzene (5aa) as a white solid in $98 \%$ yield $(0.072 \mathrm{~g}, 0.20 \mathrm{mmol}$, $E / Z=98 / 2)$.

\subsubsection{Cross-coupling reaction of 5aa with diethylzinc}

Under argon, diethylzinc (1.0 M hexane solution, $0.60 \mathrm{~mL}$, $0.60 \mathrm{mmol})$ was added to a suspension of $(E)-\{2-$ diphenylthiophosphinyl-1-(methylthio)ethenyl $\}$ benzene (5aa, $0.073 \mathrm{~g}, 0.20 \mathrm{mmol}$ ) and $\mathrm{NiCl}_{2}$ (dppp) (0.016 g, 0.030 $\mathrm{mmol})$ in CPME $(3.0 \mathrm{~mL})$ at $0{ }^{\circ} \mathrm{C}$. The reaction was monitored by TLC. After $4.5 \mathrm{~h}$, the mixture was quenched with diluted $\mathrm{NaCl}$ solution and extracted three times with EtOAc, dried over $\mathrm{Na}_{2} \mathrm{SO}_{4}$, filtered through a pad of Florisil $^{\circledR}$, and concentrated in vacuo. Silica gel column chromatography (hexane/EtOAc $=20 / 1$ to $10 / 1$ ) afforded 1diphenylthiophosphinyl-2-phenyl-1-butene (6) as a white solid in $75 \%$ yield $(0.052 \mathrm{~g}, 0.15 \mathrm{mmol}, Z / E=98 / 2)$ and 1 diphenylphosphino-2-phenyl-1-butene (7) in 14\% yield (8.7 $\mathrm{mg}, 0.027 \mathrm{mmol}, Z / E=96 / 4)$.

\subsubsection{Desulfidation of vinylphosphine sulfide 6}

Under argon, a mixture of vinylphosphine sulfide $6(0.035$ g, $0.10 \mathrm{mmol})$, tris(trimethylsilyl)silane $(0.062 \mathrm{~mL}, 0.20$ $\mathrm{mmol}$ ), and AIBN (3.2 $\mathrm{mg}, 0.020 \mathrm{mmol})$ was heated in boiling benzene $(2.0 \mathrm{~mL})$ for $4 \mathrm{~h}$. After cooled to room temperature, the solvent was removed in vacuo. Silica gel column chromatography (hexane/EtOAc $=50 / 1$ to $20 / 1$ ) afforded 1-diphenylphosphino-2-phenyl-1-butene (7) as a colorless oil in $88 \%$ yield $(0.028 \mathrm{~g}, 0.088 \mathrm{mmol}, Z / E=$ 98/2).

\subsection{Characterization Data}

\subsection{1. $S$-diphenylthiophosphinyl $O$-ethyl} dithiocarbonate (2).

IR (neat) 1436, 1366, 1254, 1096, 1033, 722, 689, $653 \mathrm{~cm}^{-}$ 1; ${ }^{1} \mathrm{H} \mathrm{NMR}\left(\mathrm{CDCl}_{3}\right) \delta 1.00(\mathrm{t}, J=7.0 \mathrm{~Hz}, 3 \mathrm{H}), 4.40(\mathrm{q}, J$ $=7.0 \mathrm{~Hz}, 2 \mathrm{H}), 7.47-7.52(\mathrm{~m}, 4 \mathrm{H}), 7.53-7.58(\mathrm{~m}, 2 \mathrm{H})$, 7.95-8.02 (m, 4H); ${ }^{13} \mathrm{C} \mathrm{NMR}\left(\mathrm{CDCl}_{3}\right) \delta 12.65,71.12$, $128.67(\mathrm{~d}, J=13.4 \mathrm{~Hz}), 131.62(\mathrm{~d}, J=11.4 \mathrm{~Hz}), 132.23$ (d, $J=2.9 \mathrm{~Hz}), 132.67$ (d, $J=84.5 \mathrm{~Hz}), 204.27$ (d, $J=4.4 \mathrm{~Hz})$; $31 \mathrm{P}$ NMR $\left(\mathrm{CDCl}_{3}\right) \delta$ 59.46. Found: $\mathrm{C}, 53.36 ; \mathrm{H}, 4.43 \%$. Calcd for $\mathrm{C}_{15} \mathrm{H}_{15} \mathrm{OPS}_{3}$ : C, 53.23; H, 4.47\%. m.p.: 58-59 ${ }^{\circ} \mathrm{C}$.

\subsection{2. (E)-S-2-diphenylthiophosphinyl-1-phenylethenyl} $O$-ethyl dithiocarbonate (3a).

IR (Nujol) 1438, 1240, 1103, 1041, 715, 693, $635 \mathrm{~cm}^{-1}$; ${ }^{1} \mathrm{H} \mathrm{NMR}\left(\mathrm{CDCl}_{3}\right) \delta 1.07(\mathrm{t}, J=7.0 \mathrm{~Hz}, 3 \mathrm{H}), 4.42(\mathrm{q}, J=$ $7.0 \mathrm{~Hz}, 2 \mathrm{H}), 6.96-7.01(\mathrm{~m}, 2 \mathrm{H}), 7.02(\mathrm{~d}, J=15.5 \mathrm{~Hz}, 1 \mathrm{H})$, 7.01-7.05 (m, 1H), 7.25-7.30 (m, 4H), 7.31-7.36 (m, 2H), 7.48-7.52 (m, 2H), 7.75-7.82 (m, 4H); ${ }^{13} \mathrm{C}$ NMR $\left(\mathrm{CDCl}_{3}\right) \delta 13.14,70.53,127.42,128.27(\mathrm{~d}, J=12.9 \mathrm{~Hz})$, $129.28,129.65,130.13(\mathrm{~d}, J=73.1 \mathrm{~Hz}), 131.27$ (d, $J=3.4$ $\mathrm{Hz}), 131.30(\mathrm{~d}, J=10.5 \mathrm{~Hz}), 132.03(\mathrm{~d}, J=86.0 \mathrm{~Hz})$, $136.44(\mathrm{~d}, J=4.8 \mathrm{~Hz}), 149.15,209.24(\mathrm{~d}, J=1.5 \mathrm{~Hz}) ;{ }^{31} \mathrm{P}$ NMR $\left(\mathrm{CDCl}_{3}\right) \delta 28.97$. Found: C, 62.74; H, 5.04\%. Calcd for $\mathrm{C}_{23} \mathrm{H}_{21} \mathrm{OPS}_{3}$ : C, 62.70; H, 4.80\%. m.p.: 106-107 ${ }^{\circ} \mathrm{C}$. The $E$ configuration was determined by X-ray analysis. Crystallographic data for the structure has been deposited with the Cambridge Crystallographic Data Centre (CCDC 710609). Copies of the data can be obtained, free of charge, on application to the Director, CCDC, 12 Union Road, Cambridge, CB2, 1EZ, UK. Fax: 44-1223-336033 or Email: deposit@ccdc.cam.ac.uk

\subsection{3. (E)-S-2-diphenylthiophosphinyl-1-(4- methylphenyl)ethenyl $O$-ethyl dithiocarbonate (3b).} IR (Nujol) 1559, 1506, 1438, 1244, 1102, 1043, 827, 715, $631 \mathrm{~cm}^{-1} ;{ }^{1} \mathrm{H} \mathrm{NMR}\left(\mathrm{CDCl}_{3}\right) \delta 1.10(\mathrm{t}, J=7.0 \mathrm{~Hz}, 3 \mathrm{H})$, $2.16(\mathrm{~s}, 3 \mathrm{H}), 4.43(\mathrm{q}, J=7.0 \mathrm{~Hz}, 2 \mathrm{H}), 6.75-6.79(\mathrm{~m}, 2 \mathrm{H})$, $6.97(\mathrm{~d}, J=16.0 \mathrm{~Hz}, 1 \mathrm{H}), 7.25-7.30(\mathrm{~m}, 4 \mathrm{H}), 7.32-7.38(\mathrm{~m}$, $4 \mathrm{H}), 7.75-7.82(\mathrm{~m}, 4 \mathrm{H}) ;{ }^{13} \mathrm{C} \mathrm{NMR}\left(\mathrm{CDCl}_{3}\right) \delta 13.20$, 21.19, 70.50, 128.03, $128.22(\mathrm{~d}, J=12.9 \mathrm{~Hz}), 129.55$ (d, $J$ $=74.0 \mathrm{~Hz}), 129.63,131.11(\mathrm{~d}, J=2.9 \mathrm{~Hz}), 131.35(\mathrm{~d}, J=$ $11.0 \mathrm{~Hz}), 132.22$ (d, $J=85.9 \mathrm{~Hz}), 133.61$ (d, $J=4.9 \mathrm{~Hz})$, 139.49, 149.27, 209.53; 31 P NMR $\left(\mathrm{CDCl}_{3}\right) \delta 29.11$. Found: C, 63.27; H, 5.07\%. Calcd for $\mathrm{C}_{24} \mathrm{H}_{23} \mathrm{OPS}_{3}$ : C, $63.41 ; \mathrm{H}, 5.10 \%$. m.p.: $123-124^{\circ} \mathrm{C}$. 
4.3.4. (E)-S-2-diphenylthiophosphinyl-1-mesitylethenyl $O$-ethyl dithiocarbonate (3c).

IR (Nujol) 1436, 1245, 1104, 1031, 852, 712, 691, $633 \mathrm{~cm}^{-}$ 1. ${ }^{1} \mathrm{H} \mathrm{NMR}\left(\mathrm{CDCl}_{3}\right) \delta 1.35(\mathrm{t}, J=7.0 \mathrm{~Hz}, 3 \mathrm{H}), 2.13(\mathrm{~s}$, $3 \mathrm{H}), 2.16(\mathrm{~s}, 6 \mathrm{H}), 4.68(\mathrm{q}, J=7.0 \mathrm{~Hz}, 2 \mathrm{H}), 6.46(\mathrm{~d}, J=0.5$ $\mathrm{Hz}, 2 \mathrm{H}), 7.24(\mathrm{~d}, J=15.5 \mathrm{~Hz}, 1 \mathrm{H}), 7.26-7.31(\mathrm{~m}, 4 \mathrm{H})$, 7.36-7.41 (m, 2H), 7.65-7.71 (m, 4H); ${ }^{13} \mathrm{C}$ NMR $\left(\mathrm{CDCl}_{3}\right) \delta 13.54,20.27,20.92,70.48,125.14$ (d, $J=78.3$ $\mathrm{Hz}), 128.05,128.06$ (d, $J=12.9 \mathrm{~Hz}), 129.97(\mathrm{~d}, J=6.3 \mathrm{~Hz})$, $131.04(\mathrm{~d}, J=2.9 \mathrm{~Hz}), 131.40(\mathrm{~d}, J=11.0 \mathrm{~Hz}), 132.41(\mathrm{~d}, J$ $=86.4 \mathrm{~Hz}), 136.33,138.72,148.99(\mathrm{~d}, J=2.9 \mathrm{~Hz}), 208.48$; $31_{\mathrm{P}} \mathrm{NMR}\left(\mathrm{CDCl}_{3}\right) \delta 29.83$. Found: $\mathrm{C}, 64.65 ; \mathrm{H}, 5.64 \%$. Calcd for $\mathrm{C}_{26} \mathrm{H}_{27} \mathrm{OPS}_{3}$ : C, 64.70; H, 5.64\%. m.p.: 93-94 ${ }^{\circ} \mathrm{C}$.

4.3.5. (E)-S-2-diphenylthiophosphinyl-1-(1naphthyl)ethenyl $\boldsymbol{O}$-ethyl dithiocarbonate (3d).

IR (Nujol) 1559, 1507, 1436, 1231, 1049, 1036, 798, 714, $633 \mathrm{~cm}^{-1} ;{ }^{1} \mathrm{H} \mathrm{NMR}\left(\mathrm{CDCl}_{3}\right) \delta 1.20(\mathrm{dd}, J=7.0,7.0 \mathrm{~Hz}$, $3 \mathrm{H}), 4.48(\mathrm{dq}, J=11.0,7.0 \mathrm{~Hz}, 1 \mathrm{H}), 4.53(\mathrm{dq}, J=11.0,7.0$ $\mathrm{Hz}, 1 \mathrm{H}), 6.75-6.80$ (m, 2H), 6.85-6.90 (m, 1H), 7.20 (dd, $J$ $=8.0,7.0 \mathrm{~Hz}, 1 \mathrm{H}), 7.26-7.32(\mathrm{~m}, 2 \mathrm{H}), 7.32-7.40(\mathrm{~m}, 3 \mathrm{H})$, $7.37(\mathrm{~d}, J=16.5 \mathrm{~Hz}, 1 \mathrm{H}), 7.41-7.47(\mathrm{~m}, 2 \mathrm{H}), 7.50-7.54(\mathrm{~m}$, $1 \mathrm{H}), 7.54-7.58(\mathrm{~m}, 1 \mathrm{H}), 7.67(\mathrm{dd}, J=7.0,1.0 \mathrm{~Hz}, 1 \mathrm{H})$, 7.73-7.80 (m, 3H); ${ }^{13} \mathrm{C} \mathrm{NMR}\left(\mathrm{CDCl}_{3}\right) \delta 13.36,70.56$, $124.26,125.40,125.84,126.11,127.15(\mathrm{~d}, J=12.9 \mathrm{~Hz})$, $128.08,128.24(\mathrm{~d}, J=12.5 \mathrm{~Hz}), 129.30(\mathrm{~d}, J=1.4 \mathrm{~Hz})$, 129.38 (d, $J=85.4 \mathrm{~Hz}), 129.89,129.93$ (d, $J=1.0 \mathrm{~Hz})$, $130.21(\mathrm{~d}, J=2.9 \mathrm{~Hz}), 130.99(\mathrm{~d}, J=11.0 \mathrm{~Hz}), 131.10(\mathrm{~d}, J$ $=10.0 \mathrm{~Hz}), 131.27(\mathrm{~d}, J=2.9 \mathrm{~Hz}), 132.53(\mathrm{~d}, J=5.8 \mathrm{~Hz})$, $132.96,133.07(\mathrm{~d}, J=86.9 \mathrm{~Hz}), 133.48(\mathrm{~d}, J=75.0 \mathrm{~Hz})$, $147.43(\mathrm{~d}, J=1.5 \mathrm{~Hz}), 208.82(\mathrm{~d}, J=1.4 \mathrm{~Hz}) ; 31_{\mathrm{P}} \mathrm{NMR}$ $\left(\mathrm{CDCl}_{3}\right) \delta 29.31$. Found: $\mathrm{C}, 66.25 ; \mathrm{H}, 4.82 \%$. Calcd for $\mathrm{C}_{27} \mathrm{H}_{23} \mathrm{OPS}_{3}$ : C, 66.10; H, 4.73\% m.p.: $115-116^{\circ} \mathrm{C}$.

\subsection{6. (E)-S-2-diphenylthiophosphinyl-1-(4-} methoxyphenyl)ethenyl $\boldsymbol{O}$-ethyl dithiocarbonate (3e). IR (Nujol) 1609, 1504, 1437, 1253, 1241, 1041, 834, 747, $712,629 \mathrm{~cm}^{-1} ;{ }^{1} \mathrm{H}$ NMR $\left(\mathrm{CDCl}_{3}\right) \delta 1.12(\mathrm{t}, J=7.0 \mathrm{~Hz}$, $3 \mathrm{H}), 3.68(\mathrm{~s}, 3 \mathrm{H}), 4.44(\mathrm{q}, J=7.0 \mathrm{~Hz}, 2 \mathrm{H}), 6.48-6.52(\mathrm{~m}$, $2 \mathrm{H}), 6.91(\mathrm{~d}, J=16.0 \mathrm{~Hz}, 1 \mathrm{H}), 7.26-7.32(\mathrm{~m}, 4 \mathrm{H}), 7.32-$ $7.37(\mathrm{~m}, 2 \mathrm{H}), 7.45-7.49(\mathrm{~m}, 2 \mathrm{H}), 7.77-7.84(\mathrm{~m}, 4 \mathrm{H}) ;{ }^{13} \mathrm{C}$ NMR $\left(\mathrm{CDCl}_{3}\right) \delta 13.28,55.23,70.49,112.81,128.26(\mathrm{~d}, J$ $=12.9 \mathrm{~Hz}), 128.72(\mathrm{~d}, J=74.0 \mathrm{~Hz}), 129.01(\mathrm{~d}, J=4.8 \mathrm{~Hz})$, $131.21(\mathrm{~d}, J=3.3 \mathrm{~Hz}), 131.35$ (d, $J=10.5 \mathrm{~Hz}), 131.39$, 132.22 (d, $J=85.5 \mathrm{~Hz}$ ), 148.88, 160.41, 209.71; ${ }^{31} \mathrm{P}$ NMR $\left(\mathrm{CDCl}_{3}\right) \delta 29.09$. Found: $\mathrm{C}, 61.25 ; \mathrm{H}, 4.98 \%$. Calcd for $\mathrm{C}_{24} \mathrm{H}_{23} \mathrm{O}_{2} \mathrm{PS}_{3}: \mathrm{C}, 61.25$; H, 4.93\%. m.p.: $105-106^{\circ} \mathrm{C}$.

4.3.7. (E)-S-1-(4-acetylphenyl)-2-

(diphenylthiophosphinyl)ethenyl $O$-ethyl dithiocarbonate (3f).

IR (Nujol) 1681, 1436, 1247, 1099, 1037, 819, 708, 627 $\mathrm{cm}^{-1} ;{ }^{1} \mathrm{H}$ NMR $\left(\mathrm{CDCl}_{3}\right) \delta 1.12(\mathrm{t}, J=7.0 \mathrm{~Hz}, 3 \mathrm{H}), 2.49$ $(\mathrm{s}, 3 \mathrm{H}), 4.43$ (q, $J=7.0 \mathrm{~Hz}, 2 \mathrm{H}), 7.13(\mathrm{~d}, J=15.5 \mathrm{~Hz}, 1 \mathrm{H})$, 7.26-7.31 (m, 4H), 7.32-7.37 (m, 2H), 7.54-7.60 (m, 4H), 7.75-7.81 (m, 4H); ${ }^{13} \mathrm{C}$ NMR $\left(\mathrm{CDCl}_{3}\right) \delta 13.25,26.62$, $70.70,127.28,128.38$ (d, $J=12.9 \mathrm{~Hz}), 129.94,131.33$ (d, $J$ $=11.0 \mathrm{~Hz}), 131.45(\mathrm{~d}, J=2.9 \mathrm{~Hz}), 131.81(\mathrm{~d}, J=86.9 \mathrm{~Hz})$, $132.67(\mathrm{~d}, J=71.6 \mathrm{~Hz}), 137.01,141.05(\mathrm{~d}, J=4.8 \mathrm{~Hz})$, $147.53,197.26,208.53 ;{ }^{3} 1_{\mathrm{P}} \mathrm{NMR}\left(\mathrm{CDCl}_{3}\right) \delta 28.73$.
Found: C, 62.35; H, 4.77\%. Calcd for $\mathrm{C}_{25} \mathrm{H}_{23} \mathrm{O}_{2} \mathrm{PS}_{3}$ : C, $62.22 ; \mathrm{H}, 4.80 \%$. m.p.: $102-103^{\circ} \mathrm{C}$.

4.3.8. (E)-S-1-(4-cyanophenyl)-2(diphenylthiophosphinyl)ethenyl $O$-ethyl dithiocarbonate (3g).

IR (Nujol) 2233, 1436, 1248, 1105, 1028, 901, 841, 716, 694, $636 \mathrm{~cm}^{-1} ;{ }^{1} \mathrm{H}$ NMR $\left(\mathrm{CDCl}_{3}\right) \delta 1.14(\mathrm{t}, J=7.0 \mathrm{~Hz}$, $3 \mathrm{H}), 4.44(\mathrm{q}, J=7.0 \mathrm{~Hz}, 2 \mathrm{H}), 7.18(\mathrm{~d}, J=15.0 \mathrm{~Hz}, 1 \mathrm{H})$, 7.26-7.30 (m, 2H), 7.30-7.35 (m, 4H), 7.37-7.42 (m, 2H), 7.58-7.62 (m, 2H), 7.74-7.80 (m, 4H); ${ }^{13} \mathrm{C}$ NMR $\left(\mathrm{CDCl}_{3}\right) \delta 13.26,70.82,112.43,118.16,128.48(\mathrm{~d}, J=$ $12.9 \mathrm{~Hz}), 130.30,131.03,131.28(\mathrm{~d}, J=10.9 \mathrm{~Hz}), 131.48$ $(\mathrm{d}, J=86.0 \mathrm{~Hz}), 131.67(\mathrm{~d}, J=2.8 \mathrm{~Hz}), 134.08(\mathrm{~d}, J=70.6$ $\mathrm{Hz}), 141.09$ (d, $J=4.8 \mathrm{~Hz}), 146.31,208.10$ (d, $J=1.5 \mathrm{~Hz}$ ); 31 P NMR $\left(\mathrm{CDCl}_{3}\right) \delta$ 28.41. Found: C, 61.97; $\mathrm{H}, 4.14 \%$. Calcd for $\mathrm{C}_{24} \mathrm{H}_{20}{ }^{\mathrm{NOPS}} 3$ : C, 61.91; H, 4.33\%. m.p.: 132$133^{\circ} \mathrm{C}$.

\subsection{9. (E)-S-2-diphenylthiophosphinyl-1-(4- methoxycarbonylphenyl)ethenyl $O$-ethyl dithiocarbonate $(3 \mathrm{~h})$.}

IR (Nujol) 1722, 1717, 1436, 1283, 1244, 1111, 1036, 707, $629 \mathrm{~cm}^{-1} ;{ }^{1} \mathrm{H} \mathrm{NMR}\left(\mathrm{CDCl}_{3}\right) \delta 1.09(\mathrm{t}, J=7.0 \mathrm{~Hz}, 3 \mathrm{H})$, $3.88(\mathrm{~s}, 3 \mathrm{H}), 4.41(\mathrm{q}, J=7.0 \mathrm{~Hz}, 2 \mathrm{H}), 7.12(\mathrm{~d}, J=15.5 \mathrm{~Hz}$, $1 \mathrm{H}), 7.26-7.32(\mathrm{~m}, 4 \mathrm{H}), 7.33-7.38(\mathrm{~m}, 2 \mathrm{H}), 7.52-7.56(\mathrm{~m}$, 2H), 7.63-7.67 (m, 2H), 7.74-7.80 (m, 4H); ${ }^{13} \mathrm{C}$ NMR $\left(\mathrm{CDCl}_{3}\right) \delta 13.22,52.17,70.67,128.38(\mathrm{~d}, J=12.9 \mathrm{~Hz})$, $128.57,129.69,130.30,131.31(\mathrm{~d}, J=10.5 \mathrm{~Hz}), 131.47$ (d, $J=2.9 \mathrm{~Hz}), 131.80(\mathrm{~d}, J=84.5 \mathrm{~Hz}), 132.31(\mathrm{~d}, J=71.6$ $\mathrm{Hz}), 140.94$ (d, $J=5.3 \mathrm{~Hz}), 147.77,166.27,208.49 ;{ }^{31} \mathrm{P}$ NMR $\left(\mathrm{CDCl}_{3}\right) \delta 28.65$. Found: C, 60.16; H, 4.75\%. Calcd for $\mathrm{C}_{25} \mathrm{H}_{23} \mathrm{O}_{3} \mathrm{PS}_{3}$ : C, $60.22 ; \mathrm{H}, 4.65 \%$. m.p.: $132-133{ }^{\circ} \mathrm{C}$.

\subsubsection{0. (E)-S-2-diphenylthiophosphinyl-1-(4-} formylphenyl)ethenyl $\boldsymbol{O}$-ethyl dithiocarbonate (3i). IR (Nujol) 1699, 1684, 1558, 1439, 1243, 1100, 1041, 825, $715 \mathrm{~cm}^{-1} ;{ }^{1} \mathrm{H} \mathrm{NMR}\left(\mathrm{CDCl}_{3}\right) \delta 1.11(\mathrm{t}, J=7.0 \mathrm{~Hz}, 3 \mathrm{H})$, $4.43(\mathrm{q}, J=7.0 \mathrm{~Hz}, 2 \mathrm{H}), 7.17(\mathrm{~d}, J=15.5 \mathrm{~Hz}, 1 \mathrm{H}), 7.26-$ $7.32(\mathrm{~m}, 4 \mathrm{H}), 7.33-7.38(\mathrm{~m}, 2 \mathrm{H}), 7.49-7.52(\mathrm{~m}, 2 \mathrm{H}), 7.64-$ $7.67(\mathrm{~m}, 2 \mathrm{H}), 7.75-7.81(\mathrm{~m}, 4 \mathrm{H}), 9.85(\mathrm{~s}, 1 \mathrm{H}) ;{ }^{13} \mathrm{C}$ NMR $\left(\mathrm{CDCl}_{3}\right) \delta 13.23,70.74,128.42(\mathrm{~d}, J=12.9 \mathrm{~Hz}), 128.60$, $130.35(\mathrm{~d}, J=1.0 \mathrm{~Hz}), 131.31(\mathrm{~d}, J=10.5 \mathrm{~Hz}), 131.54(\mathrm{~d}, J$ $=3.3 \mathrm{~Hz}), 131.69(\mathrm{~d}, J=86.0 \mathrm{~Hz}), 133.20(\mathrm{~d}, J=71.1 \mathrm{~Hz})$, $136.11,142.44(\mathrm{~d}, J=4.8 \mathrm{~Hz}), 147.23,191.39$ (d, $J=1.4$ $\mathrm{Hz}), 208.34(\mathrm{~d}, J=1.4 \mathrm{~Hz}) ;{ }^{3}{ }_{\mathrm{P}} \mathrm{NMR}\left(\mathrm{CDCl}_{3}\right) \delta 28.61$. Found: $\mathrm{C}, 61.35 ; \mathrm{H}, 4.62 \%$. Calcd for $\mathrm{C}_{24} \mathrm{H}_{21} \mathrm{O}_{2} \mathrm{PS}_{3}$ : C, $61.52 ; \mathrm{H}, 4.52 \%$. m.p.: $110-111^{\circ} \mathrm{C}$.

\subsubsection{1. (E)-S-1-(4-bromophenyl)-2- (diphenylthiophosphinyl)ethenyl $\boldsymbol{O}$-ethyl dithiocarbonate (3j).}

IR (Nujol) 1480, 1436, 1264, 1248, 1107, 1030, 819, 707 $\mathrm{cm}^{-1} ;{ }^{1} \mathrm{H} \mathrm{NMR}\left(\mathrm{CDCl}_{3}\right) \delta 1.13(\mathrm{t}, J=7.0 \mathrm{~Hz}, 3 \mathrm{H}), 4.44$ $(\mathrm{q}, J=7.0 \mathrm{~Hz}, 2 \mathrm{H}), 7.06(\mathrm{~d}, J=15.5 \mathrm{~Hz}, 1 \mathrm{H}), 7.07-7.12(\mathrm{~m}$, 2H), 7.28-7.36 (m, 6H), 7.36-7.41 (m, 2H), 7.74-7.81 (m, $4 \mathrm{H}) ;{ }^{13} \mathrm{C} \mathrm{NMR}\left(\mathrm{CDCl}_{3}\right) \delta 13.24,70.67,123.61,128.36(\mathrm{~d}$, $J=12.4 \mathrm{~Hz}), 130.50,131.18,131.34(\mathrm{~d}, J=10.5 \mathrm{~Hz})$, $131.41(\mathrm{~d}, J=2.9 \mathrm{~Hz}), 131.59(\mathrm{~d}, J=74.0 \mathrm{~Hz}), 131.75(\mathrm{~d}, J$ $=87.4 \mathrm{~Hz}), 135.44(\mathrm{~d}, J=4.8 \mathrm{~Hz}), 147.60,208.72 ;{ }^{3} \mathrm{P}$ NMR $\left(\mathrm{CDCl}_{3}\right) \delta 28.76$. Found: C, 53.04; H, 3.74\%. Calcd 
for $\mathrm{C}_{23} \mathrm{H}_{20} \mathrm{BrOPS}_{3}$ : C, 53.18; H, 3.88\%. m.p.: $118-119$ ${ }^{\circ} \mathrm{C}$.

\subsubsection{2. (E)-S-2-diphenylthiophosphinyl-1-(4-} hydroxymethylphenyl)ethenyl $O$-ethyl dithiocarbonate (3k).

IR (Nujol) 3600-3300 (br), 1654, 1506, 1436, 1242, 1041,

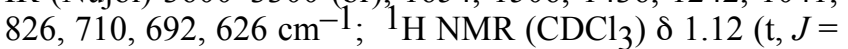
$7.0 \mathrm{~Hz}, 3 \mathrm{H}), 1.50(\mathrm{br} \mathrm{s}, 1 \mathrm{H}), 4.44(\mathrm{q}, J=7.0 \mathrm{~Hz}, 2 \mathrm{H}), 4.51$ (s, 2H), 6.96-7.00 (m, 2H), $7.03(\mathrm{~d}, J=16.0 \mathrm{~Hz}, 1 \mathrm{H})$, 7.26-7.31 (m, 4H), 7.32-7.37 (m, 2H), 7.46-7.51 (m, 2H), $7.75-7.82(\mathrm{~m}, 4 \mathrm{H}) ;{ }^{13} \mathrm{C} \mathrm{NMR}\left(\mathrm{CDCl}_{3}\right) \delta$ 13.24, 64.70, $70.56,125.73,128.28$ (d, $J=12.4 \mathrm{~Hz}), 129.94(\mathrm{~d}, J=1.0$ $\mathrm{Hz}), 130.62(\mathrm{~d}, J=73.0 \mathrm{~Hz}), 131.21(\mathrm{~d}, J=3.4 \mathrm{~Hz}), 131.33$ $(\mathrm{d}, J=10.5 \mathrm{~Hz}), 132.14(\mathrm{~d}, J=85.9 \mathrm{~Hz}), 135.78(\mathrm{~d}, J=4.8$ $\mathrm{Hz}), 142.05,148.73,209.20(\mathrm{~d}, J=1.4 \mathrm{~Hz}$ ); 31 P NMR $\left(\mathrm{CDCl}_{3}\right) \delta$ 28.97. Found: $\mathrm{C}, 61.05 ; \mathrm{H}, 4.90 \%$. Calcd for $\mathrm{C}_{24} \mathrm{H}_{23} \mathrm{O}_{2} \mathrm{PS}_{3}: \mathrm{C}, 61.25 ; \mathrm{H}, 4.93 \%$. m.p.: $111-112{ }^{\circ} \mathrm{C}$.

\subsubsection{3. $p$-bis $\{(E)$-2-diphenylthiophosphinyl-1-} (ethoxythiocarbonylsulfanyl)ethenyl\}benzene (3I).

IR (Nujol) 1577, 1559, 1436, 1246, 1098, 1041, 846, 752, $715,632 \mathrm{~cm}^{-1} ; 1_{\mathrm{H}} \mathrm{NMR}\left(\mathrm{CDCl}_{3}\right) \delta 1.39(\mathrm{t}, J=7.0 \mathrm{~Hz}$, $6 \mathrm{H}), 4.62(\mathrm{q}, J=7.0 \mathrm{~Hz}, 4 \mathrm{H}), 7.06(\mathrm{~s}, 4 \mathrm{H}), 7.10(\mathrm{~d}, J=15.5$ $\mathrm{Hz}, 2 \mathrm{H}), 7.24-7.29(\mathrm{~m}, 8 \mathrm{H}), 7.31-7.36(\mathrm{~m}, 4 \mathrm{H}), 7.71-7.78$ $(\mathrm{m}, 8 \mathrm{H}) ;{ }^{3} \mathrm{C}$ NMR $\left(\mathrm{CDCl}_{3}\right) \delta 13.70,70.96,128.34(\mathrm{~d}, J=$ $12.9 \mathrm{~Hz}), 128.77,131.29$ (d, $J=10.5 \mathrm{~Hz}), 131.54(\mathrm{~d}, J=$ $3.4 \mathrm{~Hz}), 131.86(\mathrm{~d}, J=85.9 \mathrm{~Hz}), 134.41(\mathrm{~d}, J=71.6 \mathrm{~Hz})$, $136.99(\mathrm{~d}, J=4.8 \mathrm{~Hz}), 146.75,209.85(\mathrm{~d}, J=1.9 \mathrm{~Hz}) ; 31 \mathrm{P}$ NMR $\left(\mathrm{CDCl}_{3}\right) \delta 28.41$. Found: C, 59.56; H, 4.47\%. Calcd for $\mathrm{C}_{40} \mathrm{H}_{36} \mathrm{O}_{2} \mathrm{P}_{2} \mathrm{~S}_{6}$ : C, $59.83 ; \mathrm{H}, 4.52 \%$. m.p.: $158-159$ ${ }^{\circ} \mathrm{C}$.

\subsubsection{4. (E)-\{2-diphenylthiophosphinyl-1-}

(methylthio)ethenyl $\}$ benzene (5aa).

IR (Nujol) 1684, 1653, 1558, 1540, 1507, 1437, 1095, 715, $697 \mathrm{~cm}^{-1} ;{ }^{1} \mathrm{H}$ NMR $\left(\mathrm{CDCl}_{3}\right) \delta 2.38(\mathrm{~s}, 3 \mathrm{H}), 6.05(\mathrm{~d}, J=$ $15.0 \mathrm{~Hz}, 1 \mathrm{H}), 6.97-7.01(\mathrm{~m}, 2 \mathrm{H}), 7.03-7.08(\mathrm{~m}, 1 \mathrm{H}), 7.24-$ $7.29(\mathrm{~m}, 6 \mathrm{H}), 7.29-7.34(\mathrm{~m}, 2 \mathrm{H}), 7.73-7.80(\mathrm{~m}, 4 \mathrm{H}) ;{ }^{13} \mathrm{C}$ NMR $\left(\mathrm{CDCl}_{3}\right) \delta 16.40(\mathrm{~d}, J=1.5 \mathrm{~Hz}), 112.09(\mathrm{~d}, J=88.3$ $\mathrm{Hz}), 127.59,128.08$ (d, $J=12.4 \mathrm{~Hz}), 128.91,128.99$ (d, $J=$ $1.0 \mathrm{~Hz}), 130.78(\mathrm{~d}, J=2.9 \mathrm{~Hz}), 131.31(\mathrm{~d}, J=10.5 \mathrm{~Hz})$, $133.72(\mathrm{~d}, J=85.9 \mathrm{~Hz}), 135.95$ (d, $J=6.3 \mathrm{~Hz}), 159.89$ (d, $J$ $=2.9 \mathrm{~Hz}) ;{ }^{31} \mathrm{P}$ NMR $\left(\mathrm{CDCl}_{3}\right) \delta 28.83$. Found: $\mathrm{C}, 68.55$; $\mathrm{H}, 5.25 \%$. Calcd for $\mathrm{C}_{21} \mathrm{H}_{19} \mathrm{PS}_{2}: \mathrm{C}, 68.82 ; \mathrm{H}, 5.23 \%$. m.p.: $106-107^{\circ} \mathrm{C}$. The $E$ configuration of the major isomer was determined by NOE experiments.

\subsubsection{5. (E)-\{1-allylthio-2-}

(diphenylthiophosphinyl)ethenyl $\}$ benzene (5ab).

IR (Nujol) 1558, 1539, 1507, 1437, 1096, 906, 719, 632 $\mathrm{cm}^{-1} ; 1_{\mathrm{H} \text { NMR }}\left(\mathrm{CDCl}_{3}\right) \delta 3.43$ (ddd, $J=6.5,1.0,1.0 \mathrm{~Hz}$, 2H), 5.19-5.26 (m, 2H), 5.87 (ddt, $J=17.0,10.0,6.5 \mathrm{~Hz}$, $1 \mathrm{H}), 6.25(\mathrm{~d}, J=15.5 \mathrm{~Hz}, 1 \mathrm{H}), 6.97-7.03(\mathrm{~m}, 2 \mathrm{H}), 7.03-$ $7.08(\mathrm{~m}, 1 \mathrm{H}), 7.23-7.35(\mathrm{~m}, 8 \mathrm{H}), 7.71-7.79(\mathrm{~m}, 4 \mathrm{H}) ;{ }^{13} \mathrm{C}$ NMR $\left(\mathrm{CDCl}_{3}\right) \delta 35.97,114.35(\mathrm{~d}, J=86.0 \mathrm{~Hz}), 119.04$, $127.59,128.06(\mathrm{~d}, J=12.5 \mathrm{~Hz}), 128.97,129.23$ (d, $J=0.9$ $\mathrm{Hz}), 130.80(\mathrm{~d}, J=2.9 \mathrm{~Hz}), 131.31(\mathrm{~d}, J=10.5 \mathrm{~Hz}), 131.76$, $133.65(\mathrm{~d}, J=85.9 \mathrm{~Hz}), 135.70$ (d, $J=6.3 \mathrm{~Hz}), 157.91$ (d, $J$ $=2.4 \mathrm{~Hz}) ;{ }^{31} \mathrm{P}$ NMR $\left(\mathrm{CDCl}_{3}\right) \delta 28.77$. Found: $\mathrm{C}, 70.37$;
$\mathrm{H}, 5.37 \%$. Calcd for $\mathrm{C}_{23} \mathrm{H}_{21} \mathrm{PS}_{2}$ : C, 70.38; H, 5.39\%. m.p.: $81-82{ }^{\circ} \mathrm{C}$.

4.3.16. (E)-\{1-benzylthio-2(diphenylthiophosphinyl)ethenyl\}benzene (5ac). IR (Nujol) 1653, 1558, 1540, 1507, 1437, 1097, 906, 772, $719 \mathrm{~cm}^{-1} ;{ }^{1} \mathrm{H}$ NMR $\left(\mathrm{CDCl}_{3}\right) \delta 4.05(\mathrm{~s}, 2 \mathrm{H}), 6.17(\mathrm{~d}, J=$ $15.0 \mathrm{~Hz}, 1 \mathrm{H}), 6.97-7.02(\mathrm{~m}, 2 \mathrm{H}), 7.03-7.08(\mathrm{~m}, 1 \mathrm{H}), 7.18-$ $7.23(\mathrm{~m}, 4 \mathrm{H}), 7.26-7.31(\mathrm{~m}, 4 \mathrm{H}), 7.31-7.40(\mathrm{~m}, 5 \mathrm{H}), 7.54-$ $7.60(\mathrm{~m}, 4 \mathrm{H}) ;{ }^{13} \mathrm{C}$ NMR $\left(\mathrm{CDCl}_{3}\right) \delta 35.57,114.31(\mathrm{~d}, J=$ $86.0 \mathrm{~Hz}$ ), 127.60 (two signals are overlapped), 128.03 (d, $J$ $=12.4 \mathrm{~Hz}), 128.71,128.82,128.98,129.23(\mathrm{~d}, J=0.9 \mathrm{~Hz})$, $130.71(\mathrm{~d}, J=2.8 \mathrm{~Hz}), 131.21(\mathrm{~d}, J=11.0 \mathrm{~Hz}), 133.63(\mathrm{~d}, J$ $=85.9 \mathrm{~Hz}), 135.18,135.64(\mathrm{~d}, J=6.3 \mathrm{~Hz}), 157.86(\mathrm{~d}, J=$ $2.4 \mathrm{~Hz}) ;{ }^{31} \mathrm{P}$ NMR $\left(\mathrm{CDCl}_{3}\right) \delta 28.55$. Found: $\mathrm{C}, 72.97 ; \mathrm{H}$, 5.54\%. Calcd for $\mathrm{C}_{27} \mathrm{H}_{23} \mathrm{PS}_{2}$ : C, $73.27 ; \mathrm{H}, 5.24 \%$. m.p.: $153-154^{\circ} \mathrm{C}$.

\subsubsection{4-\{(E)-2-diphenylthiophosphinyl-1-} (methylthio)ethenyl\}toluene (5ba).

IR (Nujol) 1558, 1503, 1437, 1098, 914, 825, 752, 709, 629 $\mathrm{cm}^{-1}$; ${ }^{1} \mathrm{H}$ NMR $\left(\mathrm{CDCl}_{3}\right) \delta 2.17(\mathrm{~s}, 3 \mathrm{H}), 2.36(\mathrm{~s}, 3 \mathrm{H}), 6.01$ $(\mathrm{d}, J=15.5 \mathrm{~Hz}, 1 \mathrm{H}), 6.75-6.79(\mathrm{~m}, 2 \mathrm{H}), 7.12-7.16(\mathrm{~m}, 2 \mathrm{H})$, 7.23-7.28 (m, 4H), 7.29-7.34 (m, 2H), 7.73-7.79 (m, 4H); ${ }^{13} \mathrm{C}$ NMR $\left(\mathrm{CDCl}_{3}\right) \delta 16.40(\mathrm{~d}, J=1.5 \mathrm{~Hz}), 21.14,111.77$ (d, $J=88.3 \mathrm{~Hz}), 127.99$ (d, $J=12.4 \mathrm{~Hz}), 128.22,128.89(\mathrm{~d}$, $J=1.0 \mathrm{~Hz}), 130.63(\mathrm{~d}, J=2.9 \mathrm{~Hz}), 131.34(\mathrm{~d}, J=10.5 \mathrm{~Hz})$, $133.11(\mathrm{~d}, J=6.6 \mathrm{~Hz}), 133.81(\mathrm{~d}, J=86.0 \mathrm{~Hz}), 138.88$, $160.03(\mathrm{~d}, J=2.9 \mathrm{~Hz}) ; \quad 31_{\mathrm{P}} \mathrm{NMR}\left(\mathrm{CDCl}_{3}\right) \delta 29.03$. Found: C, 69.37; H, 5.56\%. Calcd for $\mathrm{C}_{22} \mathrm{H}_{21} \mathrm{PS}_{2}$ : C, 69.44; H, 5.56\%. m.p.: $106-107^{\circ} \mathrm{C}$.

\subsubsection{8. (Z)-1-diphenylthiophosphinyl-2-phenylbutene}

(6).

IR (Nujol) 1559, 1436, 1098, 725, 712, $697 \mathrm{~cm}^{-1} ;{ }^{1} \mathrm{H}$ NMR $\left(\mathrm{CDCl}_{3}\right) \delta 1.10(\mathrm{t}, J=7.5 \mathrm{~Hz}, 3 \mathrm{H}), 2.57$ (qdd, $J=7.5$, $1.5,0.5 \mathrm{~Hz}, 2 \mathrm{H}), 6.35$ (d, $J=19.0,1.5 \mathrm{~Hz}, 1 \mathrm{H}), 6.94-7.02$ (m, 3H), 7.12-7.16 (m, 2H), 7.22-7.27 (m, 4H), 7.28-7.33 $(\mathrm{m}, 2 \mathrm{H}), 7.71-7.77(\mathrm{~m}, 4 \mathrm{H}) ;{ }^{13} \mathrm{C}$ NMR $\left(\mathrm{CDCl}_{3}\right) \delta 12.35$, $35.14(\mathrm{~d}, J=15.8 \mathrm{~Hz}), 118.82(\mathrm{~d}, J=86.8 \mathrm{~Hz}), 127.47$, 127.67, $128.01(\mathrm{~d}, J=12.4 \mathrm{~Hz}), 128.02(\mathrm{~d}, J=1.5 \mathrm{~Hz})$, $130.66(\mathrm{~d}, J=2.8 \mathrm{~Hz}), 131.26(\mathrm{~d}, J=10.5 \mathrm{~Hz}), 133.48(\mathrm{~d}, J$ $=85.4 \mathrm{~Hz}), 138.57(\mathrm{~d}, J=6.8 \mathrm{~Hz}), 162.87 ; 31 \mathrm{P} \mathrm{NMR}$ $\left(\mathrm{CDCl}_{3}\right) \delta 28.44$. Found: C, 75.96; $\mathrm{H}, 6.09 \%$. Calcd for $\mathrm{C}_{22} \mathrm{H}_{21}$ PS: C, 75.83; H, 6.08\%. m.p.: $79-80{ }^{\circ} \mathrm{C}$. The $Z$ configuration of the major isomer was determined by NOE experiments.

4.3.19. (Z)-1-diphenylphosphino-2-phenylbutene (7). IR (neat) 2966, 1585, 1478, 1433, 1095, 1026, 837, 742, $696 \mathrm{~cm}^{-1} ; 1^{1} \mathrm{H} \mathrm{NMR}\left(\mathrm{CDCl}_{3}\right) \delta 1.09(\mathrm{t}, J=7.5 \mathrm{~Hz}, 3 \mathrm{H})$, 2.60 (qdd, $J=7.5,1.5,0.5 \mathrm{~Hz}, 2 \mathrm{H}), 6.25(\mathrm{~d}, J=3.5,1.5 \mathrm{~Hz}$, 1H), 7.17-7.20 (m, 2H), 7.26-7.33 (m, 9H), 7.36-7.41 (m, $4 \mathrm{H}) ;{ }^{13} \mathrm{C}$ NMR $\left(\mathrm{CDCl}_{3}\right) \delta 13.00,33.98(\mathrm{~d}, J=6.3 \mathrm{~Hz})$, 123.41 (d, $J=7.6 \mathrm{~Hz}), 127.56,127.80,128.11,128.21$ (d, $J$ $=3.8 \mathrm{~Hz}), 128.32(\mathrm{~d}, J=6.3 \mathrm{~Hz}), 132.56(\mathrm{~d}, J=18.1 \mathrm{~Hz})$, $140.38(\mathrm{~d}, J=10.1 \mathrm{~Hz}), 141.11(\mathrm{~d}, J=7.6 \mathrm{~Hz}), 160.86$ (d, $J$ $=24.9 \mathrm{~Hz}) ; \quad 31 \mathrm{P}$ NMR $\left(\mathrm{CDCl}_{3}\right) \delta-28.20$. Calcd for $\mathrm{C}_{22} \mathrm{H}_{21}$ PS: C, 83.52; H, 6.69\%. HRMS Found: 316.1382 $(\Delta=+0.2 \mathrm{ppm})$; calcd for $\mathrm{C}_{22} \mathrm{H}_{21} \mathrm{P}: 316.1381[\mathrm{M}]^{+}$. The 
$Z$ configuration of the major isomer was determined by NOE experiments.

\section{Ackknowledgement}

This work is supported by Grants-in-Aid for Scientific Research and GCOE Research from the Ministry of Education, Culture, Sports, Science, and Technology, Japan. A.S. acknowledges JSPS for financial support.

\section{References}

1. Reviews: (a) Ogawa, A. J. Synth. Org. Chem., Jpn. 1995, 53, 869-880. (b) Ogawa, A.; Hirao, T. Rev. Heteroatom Chem. 1998, 18, 1-10. (c) Renaud, P. Top. Curr. Chem. 2000, 208, 81-112.

2. (a) Murphy, P. J. In Organophosphorus Reagents; Murphy, P J., Ed.; Oxford University Press: New York; 2004, Chapter 1. (b) Quin, L. D. Guide to Organophosphorus Chemistry; John Wiley \& Sons: New York; 2000.

3. (a) Tzschach, V. A.; Baensch, S. J. Prakt. Chem. 1971, 313 , 254-258. (b) Morse, J. G.; Mielcarek, J. J. J. Fluorine Chem. 1988, 40, 41-49. (c) Kawaguchi, S.; Nagata, S.; Shirai, T.; Tsuchii, K.; Nomoto, A.; Ogawa, A. Tetrahedron Lett. 2006, 47, 3919-3922. (d) Carta, P.; Puljic, N.; Robert, C.; Dhimane, A.-L.; Fensterbank, L.; Lacôte, E.; Malacria, M. Org. Lett. 2007, 9, 1061-1063. (e) Shirai, T.; Kawaguchi, S.; Nomoto, A.; Ogawa, A. Tetrahedron Lett. 2008, 49, 4043-4046.

4. (a) Sato, A.; Yorimitsu, H.; Oshima, K. Angew. Chem. Int. Ed. 2005, 44, 1694-1696. (b) Wada, T.; Kondoh, A.; Yorimitsu, H.; Oshima, K. Org. Lett. 2008, 10, 1155-1157.

5. Zard and co-workers recently reported many radical reactions using dithiocarbonates. See: (a) Zard, S. Z. Angew. Chem. Int Ed. Engl. 1997, 36, 672-685. (b) Quiclet-Sire, B.; Zard, S. Z. Phosphorus Sulfur Silicon Relat. Elem. 1999, 153, 137-145. (c) Zard, S. Z. In Radicals in Organic Synthesis; Renaud, P.,
Sibi, M. P., Eds.; Wiley-VCH: Weinheim, 2001; Vol. 1, pp 90-108. (b) Quiclet-Sire, B.; Zard, S. Z. Top. Curr. Chem. 2006, 264, 201-236. (e) Quiclet-Sire, B.; Zard, S. Z. Chem. Eur. J. 2006, 12, 6002-6016. (f) Zard, S. Z. Org. Biomol. Chem. 2007, 5, 205-213. (g) Corbet, M.; Ferjancic, Z.; Quiclet-Sire, B.; Zard, S. Z. Org. Lett. 2008, 10, 3579-3582.

6. Some examples to synthesize $Z$ isomers were reported. See: (a) Han, L.-B.; Tanaka, M. Chem. Lett. 1999, 863-864. (b) Braga, A. L.; Alves, E. F.; Silveira, C. C.; de Andrade, L. H. Tetrahedron Lett. 2000, 41, 161-163. (c) Kondoh, A.; Yorimitsu, H.; Oshima, K. Org. Lett. 2007, 9, 1383-1385.

7. The use of AIBN as a radical initiator instead of DLP worked as well.

8. GPC, which can separate a mixture by sizes of molecules, was used when silica gel column chromatography could not separate $\mathbf{3}$ from $\mathbf{2}$.

9. Parsons, A. F.; Sharpe, D. J.; Taylor, P. Synlett 2005, 29812983.

10. Sato, A.; Yorimitsu, H.; Oshima, K. Chem. Asian J. 2007, 2 , $1568-1573$

11. Thiophosphinylated vinyl sulfides $\mathbf{5}$ are gradually isomerized overnight to a 1:1 mixture of $E / Z$ isomers in $\mathrm{CDCl}_{3}$.

12. (a) Dubbaka, S. R.; Vogel, P. Angew. Chem. Int. Ed. 2005, 44, 7674-7684; and references cited therein. (b) Kanemura, S.; Kondoh, A.; Yorimitsu, H.; Oshima, K. Synthesis 2008, $2659-2664$.

13. Romeo, R.; Wozniak, L. A.; Chatgilialoglu, C. Tetrahedron Lett. 2000, 41, 9899-9902.

14. (a) Meijer, J.; Westmijze, H.; Vermeer, P. Recl. Trav. Chim. Pays-Bas 1976, 95, 102-104. (b) Kanemura, S.; Kondoh, A.; Yorimitsu, H.; Oshima, K. Org. Lett. 2007, 9, 2031-2033.

\section{Supplementary Material}

NOE spectra of 5aa, 6, and 7. Supplementary data associated with this article can be found in the online version, at doi: 10.1016/j.tet.2008.12.071. 